\title{
Design Thinking na Reestruturação do Sistema de Avaliação de Disciplina em um Curso de Medicina
}

\section{Design Thinking in Restructuring the Assessment System of a Medical Course Discipline}

\author{
João Carlos de Souza Côrtes Júnior ${ }^{I}\left(\left[-{ }_{0}\right.\right.$ \\ Maria Cristina Almeida de Souza ${ }^{I}$ (iD \\ Marlon Mohamud Vilagra ${ }^{I}(\mathbb{D}$ \\ Paula Pitta de Resende Côrtes ${ }^{I}$ (iD \\ Sandra Maria Barroso Werneck Vilagra ${ }^{I}$ iD \\ Patrícia Zen Tempski ${ }^{I I}$
}

\section{PALAVRAS-CHAVE}

- Criatividade.

- Educação Médica.

- Empatia
IUniversidade de Vassouras, Vassouras, Rio de Janeiro, Brasil.

${ }^{\mathrm{II}}$ Universidade de São Paulo, São Paulo, São Paulo, Brasil. 


\section{KEYWORDS}

- Creativity.

- Medical Education.

- Empathy.

Recebido em 30/4/20

Aceito em 23/7/20

\section{ABSTRACT}

Introduction: Innovative, student-centered methodologies help knowledge construction, allowing students to participate in developing proposed solutions for real problems. Design thinking (DT) is a process that searches for complex problems solutions in an empathetic, collaborative, and creative way. In our report, DT was applied to medical education, as a strategy for the academic community to participate directly qualifying the training. Method: We used the DT process in an experience report to restructure the assessment system used in a discipline of a medical course. Results: A shared and collaborative form of prototyping allowed for the identification of solutions that resulted in optimized quality of the assessment process of the discipline, with the adoption of new methods. Conclusions: DT applied to the field of medical education allows one to reflect on and operationalize an innovative process in the restructuring of the evaluation of the programmatic content of a discipline in the undergraduate medical course.

\section{INTRODUÇÃO}

O design thinking (DT) é um processo que propõe a busca, de forma empática, colaborativa e criativa, de soluções para problemas complexos ${ }^{1}$. A proposta do DT foi popularizada por David M. Kelley, fundador da Ideo, empresa de design e inovaçãón ${ }^{2}$, que originalmente propôs o modelo composto por três etapas: inspiração, ideação e implementação. A inspiração representa o problema que motiva a busca por soluções, a ideação remete ao processo de gerar e testar ideias, e a implementação refere-se ao caminho de tornar o projeto realidade ${ }^{3}$. Há diferentes modelos de DT, como o do diamante duplo, proposto pela British Design Council, que é composto por quatro etapas (descobrir, definir, desenvolver e entregar) e definido como o constante processo de expandir e refinar ideias nos projetos ${ }^{4}$.

Segundo os designers, o DT pode ser aprendido e aplicado por qualquer pessoa, para fins comerciais, sociais e educacionais ${ }^{5}$. Representa um processo que, pautado na empatia ${ }^{4,6}$, pode ser empregado na busca por soluções inovadoras, demandando dos que o utilizam um olhar holístico, multidisciplinar, com foco no usuário final do produto ${ }^{7}$.

Caracterizado por uma abordagem usuário-centrada, alinhada ao paradigma construtivista, capaz de valorizar a visão multidisciplinar dos participantes e empregar o processo colaborativo, o DT pode contribuir para a solução de problemas complexos na educação médica, embora sejam escassos os relatos com sua utilização nessa área ${ }^{8}$.

A solução de problemas educacionais complexos, da reforma curricular à melhoria do ambiente de aprendizado clínico, requer pensamento criativo para gerar soluções inovadoras ${ }^{1}$. Na educação médica, o método da abordagem pelo design - ao viabilizar prototipagem de ideias - pode contribuir para a resolução de problemas na área da saúde pelos estudantes, mesmo no estágio inicial de sua formação, oportunizando-lhes a prática da empatia e das habilidades necessárias ao exercício profissional'.

A ruptura tecnológica na medicina está forçando a educação médica a passar por uma transformação que exige a consideração, pelos estudantes, dos princípios de design para fornecer melhores experiências para pacientes e para a equipe médica. O programa de dispositivos médicos da Universidade Thomas Jefferson da Filadélfia, no Estados Unidos, promove a reunião de estudantes e professores em equipes, que apresentam ideias para problemas a serem enfrentados. O público seleciona ideias dignas de financiamento e as equipes começam a prototipar, revelando aplicabilidade do $\mathrm{DT}^{10}$.

O DT deve ser pensado de forma não linear, já que a presença de distintos olhares humaniza o processo de inovação por meio da empatia, criando soluções de pessoas para pessoas e com pessoas ${ }^{11}$. Surgem então novas etapas no DT: descoberta, interpretação, ideação, experimentação e evolução ${ }^{12}$.

O DT se aplica, portanto, à educação médica, na medida em que representa um valioso recurso para que a comunidade acadêmica participe diretamente da qualificação do ensino a partir da identificação de problemas complexos, bem como da elaboração de ideias para solucionálos, da implementação das soluções propostas e da avaliação do alcance dos objetivos por meio da análise de seus impactos. Entende-se por problema complexo aquele que não possui apenas uma única solução ou uma solução definitiva. Assim, diante de um problema que não tenha soluções simples, para o qual não haja métricas ou informações suficientes para resolvê-lo, o educador pode usar o DT para quebrar barreiras e gerar novos insights ${ }^{13}$.

O objetivo deste artigo é relatar a experiência com a utilização do DT na reestruturação do sistema de avaliação de uma disciplina do curso de graduação em Medicina da Universidade de Vassouras, localizada no Estado do Rio de Janeiro.

\section{RELATO DE EXPERIÊNCIA}

O curso de Medicina da Universidade de Vassouras tem, entre seus diferenciais, a dinamicidade do projeto pedagógico ${ }^{14}$, estruturado de acordo com as Diretrizes Curriculares Nacionais para os Cursos de Graduação em Medicina ${ }^{15}$. A fim de otimizarem o alcance dos objetivos do curso, os gestores instituíram ações como a disponibilização de diversos canais de comunicação aos atores sociais.

A experiência teve início com a escuta viabilizada por meio de um desses canais: o G10. Cada período do curso tem um G10, grupo constituído por dez alunos, cuja função, entre tantas outras, é dar voz às demandas dos estudantes. O G10 do quarto período, em que está alocada a disciplina Prática de Integração Ensino-Serviço-Comunidade (Piesc), cuja carga horária se destina à abordagem do conteúdo Sistema Único de Saúde, sinalizou aos gestores a insatisfação com a prova prática, que não correspondia às expectativas, por causa da repetição do conteúdo teórico, não lhes oportunizando o exercício da criatividade. Os integrantes do G10, em uma demonstração da vontade de participar da implementação de propostas inovadoras, sugeriram recursos e estratégias que poderiam viabilizar um novo modelo de prova prática.

REVISTA BRASILEIRA DE EDUCAÇÃO MÉDICA

2 44 (4) : e118; 2020 
Sensibilizados, os professores aceitaram desafio de melhorar a prova pela adoção do DT, desde que houvesse concordância dos estudantes, bem como se realizasse, pós- implementação, análise dos impactos. Segundo os gestores, a vontade dos estudantes em serem partícipes na implementação das inovações já havia sido explicitada pelo G10. Compreendida a proposta do DT, com suporte dos membros do Núcleo Docente Estruturante (NDE) - multiplicadores do conteúdo aprendido no curso de pós-graduação em Educação na Saúde ofertado pelo Centro de Desenvolvimento de Educação Médica (Cedem) da Universidade de São Paulo - e com acesso ao referencial teórico, a equipe iniciou o DT.

O DT foi realizado em cinco etapas: descoberta, interpretação, ideação, experimentação e evolução ${ }^{1}$. Como a primeira dessas etapas aconteceu no momento em que os estudantes informaram o problema, partiu-se para a segunda, a interpretação, quando se verificaram o ponto de vista dos envolvidos e suas necessidades. Os estudantes foram convidados a relatar sua percepção sobre a prova prática, a forma como vinha sendo realizada, o que permitiu verificar a empatia entre os participantes e docentes, condição imprescindível à realização do DT. Dessa forma, docentes e estudantes compartilharam suas percepções para as quais não houve qualquer tipo de julgamento (um dos requisitos do DT) a fim de não inibir o surgimento de ideias criativas. Aos presentes foi dada a liberdade para expressarem suas angústias e expectativas relacionadas ao problema, que foram então registradas a fim de subsidiarem a terceira etapa, denominada ideação. Essa etapa teve início com uma tempestade cerebral (brainstorm), que, como geradora de sugestões, viabilizou o afloramento da criatividade dos participantes. Converteram-se os dados em propostas, que foram categorizadas a fim de que fosse possível avaliar a prevalência, a factibilidade e ainda a capacidade delas de despertar o interesse do estudante em realizá-las como prova prática.

Três propostas de avaliação prática foram pré-selecionadas pelos estudantes para representação da nota: 1. assiduidade às unidades de saúde durante as visitas; 2 . apresentação do conteúdo de artigo científico relacionado ao conteúdo da disciplina; 3. estruturação da rede de atenção à saúde (RAS) de um fictício município, cujos dados fossem fornecidos na ocasião da avaliação. Foi ratificado que a realização da segunda e da terceira proposta seria em grupo e necessitaria ser apresentada aos professores, fortalecendo o exercício das competências comunicação e interação. Após análise da viabilidade das propostas, em votação aberta, elegeu-se a terceira, que foi implementada no bimestre seguinte, caracterizando a fase de evolução, também conhecida como experimentação ou prototipagem. A prova prática, até então constituída por perguntas escritas para serem respondidas sobre aspectos da visita às unidades de saúde, adquiriu um novo formato.

Para a operacionalização da prova prática, foram organizados oito grupos com seis estudantes, cuja composição atendeu à ordenação alfabética. Os participantes receberam dados do fictício município "Guaritá". Tiveram também a informação de que desempenhariam o papel de gestores públicos do município, aos quais foi delegada a responsabilidade pela elaboração da RAS. Após, os grupos deveriam apresentar a proposta aos pares e aos professores. Por meio de um checklist, os docentes avaliavam os serviços propostos por cada grupo e registravam a nota obtida, cujo valor compôs, com a nota teórica, a média bimestral. Um feedback sobre o desempenho do grupo foi dado aos estudantes. A nota obtida era a mesma para todos os integrantes do grupo a fim de que percebessem a relevância da colaboração e da participação de todos para o sucesso da atividade. A prototipagem da proposta teve momentos de descontração aliados aos de construção de conhecimento, que, de forma lúdica e criativa, mostrou-se relevante à graduação em Medicina.

A quinta e última etapa foi a avaliação da inovação, quando os participantes responderam a um instrumento sobre o alcance do objetivo. A análise dos resultados revelou que a inovação foi válida, útil e pode ser reproduzida. O novo modelo de prova prática foi muito bem avaliado pelos envolvidos, tendo alcançado $99 \%$ de aprovação. Feedback foi dado pela equipe docente ao G10, aos gestores acadêmicos e também aos membros do NDE sobre o êxito da inovação.

Os estudantes da atualidade, nativos digitais, têm expectativas por protagonizarem o processo de ensino-aprendizagem. Unidos na globalização pela tecnologia, esses jovens diferem-se daqueles de gerações pregressas na medida em que buscam a identificação com a função que desempenham e o equilíbrio entre a vida pessoal e profissional ${ }^{16,17}$. Na área educacional, têm expectativa por inovações e por aquilo que é capaz de gerar impacto positivo no seu dia a dia. Despertar o interesse desses jovens demanda a adoção de inovações metodológicas, capazes de fomentar sua participação e autonomia. Torna-se necessário que o professor procure por um novo fazer pedagógico no qual a sala de aula seja um espaço de interação e de colaboração, condição em que o DT pode ser útil e aplicável ${ }^{18}$.

Essa comunicação sistematizada, ao potencializar a empatia entre os atores sociais, contribuiu para que os gestores propusessem adoção do DT, que poderia impactar positivamente o cotidiano pedagógico, pois possuía como um dos diferenciais ser usuário-centrado, indo ao encontro dos objetivos do curso ${ }^{1,18}$. A adoção do DT pelos gestores se balizou na premissa de que a inovação pode ser aplicada em qualquer situação que demande colocar o ser humano no centro do processo, para que se possam com isso descobrir soluções criativas para os problemas complexos.

A sensibilidade dos gestores em identificar uma situação-problema complexa e a ciência de que um dos objetivos do DT é desenvolver um processo intencional para chegar a soluções colaborativas e criar impacto positivo $^{1,12}$ nortearam a busca de soluções criativas para o problema. Os gestores perceberam que os pré-requisitos para implementação do DT, como empatia, colaboração, cooperação, otimismo, inexistência de ideias preconcebidas e disposição para aceitar o novo ${ }^{1,12,18}$, faziam-se presentes, além da existência de equipe multidisciplinar, cuja variedade de percepções seria imprescindível para o êxito.

A conviç̧ão dos gestores de que a abordagem inovadora poderia ser adotada se deveu à inexistência da ideia preconcebida de que educadores devem sempre ser modelos perfeitos. Ou seja, estavam cientes de que esse tipo de expectativa dificulta assumir riscos e limita as possibilidades de criar mudanças. Educadores precisam ter também a oportunidade de experimentar, e o DT permite aprender fazendo ${ }^{12}$. Assim, o DT oportuniza aos participantes a chance de aprender com eventuais erros porque deles surgem novas ideias, de modo a viabilizar a reconsideração dos pensamentos iniciais.

$\mathrm{Na}$ experiência descrita, a empatia perpassou todo o processo, iniciando-se pelo compartilhamento das demandas dos alunos do G10 com os gestores, cuja ousadia em propor e implementar o DT esteve norteada pelo compromisso com a qualidade do ensino e com as necessidades dos estudantes. Coube aos docentes aceitar o desafio de inovar na realização da prova prática, assim como dirimir dúvidas que surgiram durante a

REVISTA BRASILEIRA DE EDUCAÇ̃̃o MÉDICA

3 44 (4) : e118; 2020 
experiência. Mas o otimismo dos estudantes e a expectativa de que poderiam participar da construção de um novo modelo de prova prática foram, sem dúvida, elementos propulsores para que a implementação tivesse todas as etapas realizadas.

\section{CONCLUSÕES}

O DT revelou-se um processo que pode ser utilizado por diferentes atores sociais no processo educacional, auxiliando-os a se estruturar para resolverem problemas de forma colaborativa, criativa e pensada para o usuário final. O DT contribuiu para o desenvolvimento de uma cultura de inovação na instituição e estimulou a empatia e a utilização do conhecimento para criar oportunidades, realizar experiências, testar propostas e avaliar resultados por meio de feedback e de avaliação do impacto.

Conclui-se que o DT pode vir a ser um aliado na inovação do processo pedagógico na educação médica, cujas soluções construídas de maneira compartilhada e colaborativa se dão a partir do entendimento do problema no contexto em que foi identificado. As diferentes visões e percepções dos participantes foram fundamentais para o êxito da experiência.

A experiência da aplicação do DT na reestruturação do sistema de avaliação de uma disciplina representou um desafio, assim como se constituiu em uma oportunidade de inovar usando uma ferramenta para a qualificação do processo avaliativo. Os alunos partícipes sentiram-se valorizados e contribuíram para a resolução do problema por eles definido.

A realização do DT permitiu pensar e operacionalizar um processo inovador na reestruturação da avaliação do conteúdo programático de uma disciplina no curso de graduação em Medicina.

\section{REFERENNCIAS}

1. Gottlieb M, Wagner E, Wagner A, Chan T. Applying design thinking principles to curricular development in medical education. AEM Education and Training. 2017;1(1):21-6 [acesso em 2 jan 2020]. Disponível em: https://www.ncbi.nlm.nih.gov/pmc/articles/ PMC6001598/.

2. Significado de design thinking. Significados; 2016 [acesso em 2 jan 2020]. Disponível em: https://www.significados.com.br/design-thinking/.

3. Brown T, Wyatt J. Design thinking for social innovation. Stanford Social Innovation Review. 2010:31-35.

4. Duarte AR. Design thinking na educação. Anais do $13^{\circ}$ Congresso Brasileiro de Pesquisa e Desenvolvimento em Design; 2018; São Paulo. São Paulo: Blucher; 2019 p. 763-777 [acesso em 30 dez 2019]. Disponível em: https://www.proceedings.blucher.com.br/articledetails/design-thinking-na-educao-29956.

5. Brown T. Design thinking: uma poderosa metodologia para decretar o fim das velhas ideias. Rio de Janeiro: Elsevier; 2010.

6. Lockwood T. Design thinking: integrating innovation, customer experience, and brand value. New York: Skyhorse; 2010.

7. Fratin RL. Design thinking aplicado à educação [dissertação]. São Paulo: Universidade Presbiteriana Mackenzie; 2016 [acesso em 2 jan 2020]. Disponível em: http://tede.mackenzie.br/jspui/handle/tede/2946.

8. McLaughlin JE, Wolcott MD, Hubbard D, Umstead K, Rider TR. A qualitative review of the design thinking framework in in health professions education. BMC Med Educ. 2019;19(1):2-8 [acesso em 5 jun 2020]. Disponível em: https://pubmed.ncbi.nlm.nih. gov/30947748/.

9. Albala L, Bober T, Mallozzi M, Koeneke-Hernandez L, Ku B. Designthinking, making, and innovating: fresh tools for the physician's toolbox. Universal Journal of Educational Research. 2018; 6(1):17983 [acesso em 5 jun 2020]. Disponível em: http://www.hrpub.org/ download/20171230/UJER18-19510091.pdf.

10. McCormick D. Using design principles to reshape medical education. The Chamber of Commerce for Greater Philadelphia, CEO Council for Growth. 2020. [acesso em 30 jul 2020]. Disponível em: https:// newphiladelphiacasebook.com/using-design-principles-to-reshapemedical-education.html.

11. Bacich L, Moran. J., organizadores. Metodologias ativas para uma educação inovadora: uma abordagem teórico-prática. Porto Alegre: Penso, 2018.

12. Design thinking para educadores [acesso em 2 jan 2020]. Disponível em: http://www.dtparaeducadores.org.br/site/.

13. Keely D, Keeley T. Creative confidence. New York: Crown; 2013.

14. Universidade de Vassouras. Projeto pedagógico do curso de Medicina. Vassouras: Universidade de Vassouras 2019 [acesso em 3 jan 2020]. Disponível em https://www.universidadedevassouras.edu.br/arquivos/ graduacao/medicina/2019-1/PPC_MEDICINA.pdf.

15. Brasil. Diretrizes Curriculares Nacionais para Cursos de Graduação em Medicina [acesso em 3 jan 2020]. Disponível em: http://portal. mec.gov.br/cne/arquivos/pdf/Med.pdf.

16. Carvalho NCO. Millennials: quem são e o que anseiam os jovens da geração Y [monografia]. Rio de Janeiro: Universidade Federal do Rio de Janeiro; 2017 [acesso em 5 jan 2020]. Disponível em: https:// pantheon.ufrj.br/bitstream/11422/4865/1/Monografia\%20-\%20 Nathalia\%20Carvalho.pdf.

17. Prensky M. Digital natives digital immigrants. On the HORIZON. NCB University Press. 2001;9(5): 1-6 [acesso em 20 mar 2020]. Disponível em: https://www.marcprensky.com/writing/Prensky\%20-\%20Digital\%20 Natives,\%20Digital\%20Immigrants\%20-\%20Part1.pdf.

18. Machado $A B$, Silva $A R L$. Intersecções entre design thinkings $e$ formação de professores. R Eletr do Alto Vale do Itajaí. 2017;6(9):7786 [acesso em 4 jan 2020]. Disponível em: http://www.revistas.udesc. br/index.php/reavi/article/view/2316419006092017077.

\section{CONTRIBUIÇÃO DOS AUTORES}

Todos os autores participaram ativamente da redação do artigo, da revisão e aprovação da versão final.

\section{CONFLITO DE INTERESSES}

Os autores declaram não haver conflito de interesses neste estudo.

\section{ENDEREÇO PARA CORRESPONDÊNCIA}

Maria Cristina Almeida de Souza. Avenida Expedicionário Oswaldo Almeida Ramos, 280, Centro, Vassouras, RJ, Brasil. CEP: 27700-000.

E-mail:mcas.souza@uol.com.br 